\title{
Effectiveness of intrapartum fetal surveillance to improve maternal and neonatal outcomes: a systematic review and network meta-analysis
}

\author{
Bassel H. Al Wattar PhD, Emma Honess MBChB, Sarah Bunnewell MBChB, Nicky J. Welton PhD, \\ Siobhan Quenby PhD, Khalid S. Khan MSc, Javier Zamora PhD, Shakila Thangaratinam PhD
}

Cite as: CMAJ 2021 April 6;193:E468-77. doi: 10.1503/cmaj.202538

\begin{abstract}
BACKGROUND: Cesarean delivery is the most common surgical procedure worldwide. Intrapartum fetal surveillance is routinely offered to improve neonatal outcomes, but the effects of different methods on the risk of emergency cesarean deliveries remains uncertain. We conducted a systematic review and network meta-analysis to evaluate the effectiveness of different types of fetal surveillance.
\end{abstract}

METHODS: We searched MEDLINE, Embase and CENTRAL until June 1, 2020, for randomized trials evaluating any intrapartum fetal surveillance method. We performed a network meta-analysis within a frequentist framework. We assessed the quality and network inconsistency of trials. We reported primarily on intrapartum emergency cesarean deliveries and other secondary maternal and neonatal outcomes using risk ratios (RRs) and 95\% confidence intervals (Cls).

RESULTS: We included 33 trials (118 863 patients) evaluating intermittent auscultation with Pinard stethoscope/handheld Doppler (IA), cardiotocography (CTG), computerized cardiotocography (cCTG), CTG with fetal scalp lactate (CTG-lactate), CTG with fetal scalp pH analysis (CTG-FBS), CTG with fetal pulse oximetry (FPO-CTG), CTG with fetal heart electrocardiogram (CTG-STAN) and their combinations. Intermittent auscultation reduced the risk of emergency cesarean deliveries compared with other types of surveillance (IA v. CTG: RR 0.83, 95\% Cl 0.72-0.97; IA v.
CTG-FBS: RR 0.71, 95\% Cl 0.63-0.80; IA v.CTG-lactate: RR $0.77,95 \% \mathrm{Cl} 0.64-0.92$; IA v. FPO-CTG: RR 0.75, 95\% Cl 0.65-0.87; IA v.FPO-CTG-FBS: RR $0.81,95 \% \mathrm{Cl} 0.67-$ 0.99; cCTG-FBS v. IA: RR $1.21,95 \% \mathrm{CI}$ 1.04-1.42), except STAN-CTG-FBS (RR 1.17, 95\% Cl 0.98-1.40). There was a similar reduction observed for emergency cesarean deliveries for fetal distress. None of the evaluated methods was associated with a reduced risk of neonatal acidemia, neonatal unit admissions, Apgar scores or perinatal death.

INTERPRETATION: Compared with other types of fetal surveillance, intermittent auscultation seems to reduce emergency cesarean deliveries in labour without increasing adverse neonatal and maternal outcomes. esarean delivery is the most common surgical procedure worldwide, performed to expedite delivery and avoid neonatal complications. Fetal surveillance is routinely offered to patients in labour to reduce the risk of adverse neonatal outcomes, as well as the risk of unnecessary emergency cesarean deliveries and other maternal interventions. Fetal surveillance aims to detect intrapartum hypoxia from acute or subacute adverse events in labour requiring medical intervention and to reduce the risk of serious complications such as neonatal cerebral palsy, hypoxic-ischemic encephalopathy or stillbirth.

Monitoring the fetal heart rate to detect intrapartum hypoxia using simple surveillance techniques, such as the Pinard stethoscope, has been practised for decades. ${ }^{1}$ Over the last 50 years, several newer surveillance methods have been evaluated, with varied uptake in practice. ${ }^{2}$ Cardiotocography (CTG) remains the most common surveillance method used in high-risk pregnancies. ${ }^{3}$ However, given its limited accuracy, many researchers have evaluated its use in combination with other surveillance methods, such as fetal heart electrocardiogram (STAN), fetal scalp $\mathrm{pH}$ analysis (FBS) or fetal pulse oximetry (FPO), and with computer-aided decision models (CCTG) to improve its diagnostic value. ${ }^{4-6}$ Despite extensive investment in clinical research, the overall effectiveness of such methods in improving maternal and neonatal outcomes remains debatable as stillbirth rates have plateaued worldwide, while cesarean delivery rates continue to rise. ${ }^{7,8}$ 
Existing meta-analyses evaluating different intrapartum fetal surveillance methods remain limited to head-to-head comparisons of monitoring techniques, without a comprehensive assessment of their effectiveness in improving maternal and neonatal pregnancy outcomes. ${ }^{4,9-13}$ We conducted a systematic review of randomized trials and a network meta-analysis to evaluate the effectiveness of all available fetal surveillance methods in improving maternal and neonatal outcomes.

\section{Methods}

\section{Search strategy}

We conducted our systematic review according to a prospectively registered protocol (CRD42018118082) and reported the findings as per established guidelines. ${ }^{14}$ We searched MEDLINE, Embase and Cochrane CENTRAL from database inception to June 1, 2020, for randomized trials evaluating any fetal surveillance method in labour. We developed our search strategy by consulting relevant Medical Subject Headings (MeSH) and combining key search words using Boolean operators within the NICE Healthcare Databases Advanced Search platform (hdas.nice.org. uk) (Appendix 1, available at www.cmaj.ca/lookup/doi/10.1503/ cmaj.202538/tab-related-content). We also conducted supplementary searches in Google Scholar and Scopus to identify any missing evidence. No search filters or language limitations were employed; articles in non-English languages were obtained and translated if deemed relevant. We manually screened the bibliographies of potentially relevant articles and published systematic reviews on the topic to identify any additional relevant trials. We contacted the authors of included trials seeking further information as necessary. We did not include any unpublished data in the analysis. We consulted the James Lind Library and previous Cochrane reviews to identify the primary outcome and other outcomes of interest to stakeholders. We did not involve patients in the design of our research study.

\section{Selection of studies and outcome reporting}

Two reviewers (E.M. and S.B.) independently selected and performed data extraction, which was further checked by a third reviewer (B.H.A.). Inconsistencies and disagreements were resolved by discussion and consensus with a fourth reviewer (S.T.). We included all randomized controlled trials that evaluated the effectiveness of existing fetal surveillance methods in labour. We included studies with multiple comparison arms, those evaluating a combination of surveillance types (e.g., fetal electrocardiogram and cardiotocography) and studies involving both high- and low-risk pregnancies. We combined instruments used for the same surveillance type under the same comparison arm (e.g., Pinard stethoscope and hand-held Doppler combined as intermittent auscultation). We excluded studies that included only preterm pregnancies and those comparing different instruments for the same surveillance type (e.g., Pinard stethoscope v. hand-held Doppler for intermittent auscultation). We also excluded studies that did not report on any of the outcomes we set a priori in our protocol (e.g., studies reporting only on the number of fetal blood sampling attempts).
Our main outcome was the incidence of emergency cesarean deliveries in labour. We also reported on other maternal outcomes such as emergency cesarean delivery in labour specifically for suspected fetal distress, instrumental deliveries and instrumental deliveries specifically for suspected fetal distress. Neonatal outcomes included neonatal acidemia (diagnosed using umbilical cord blood gases, as defined by the study authors), admissions to the neonatal intensive care unit, neonatal Apgar scores $<7$ at the 5th minute and perinatal death (i.e., any fetal death beyond 24 weeks' gestation, plus any neonatal death within the first week of life). We extracted data on the country of the study, the publication journal, trial settings, population characteristics, the evaluated fetal surveillance types, the criteria used to interpret the surveillance findings, the action algorithm used in the trial setting for acting on worrying fetal signs and the definition of fetal acidemia used by the trial investigators.

\section{Quality assessment}

Two reviewers (E.H. and S.B.) independently assessed the quality of included studies using the Cochrane risk of bias assessment tool. ${ }^{15}$ Each study was assessed for the quality of randomization and sequence generation, assignment concealment, outcome assessment, completeness of outcome data and selective outcome reporting. Blinding was deemed nonfeasible because of the nature of the evaluated interventions and was excluded from the quality assessment. Efforts to standardize the management of patients with suspected fetal distress were taken into account when evaluating detection and outcome assessment bias. Similarly, trials that employed a quality assurance process to ensure adequate diagnosis of fetal compromise against their study protocol were deemed to have less risk of outcome assessment bias compared with those that did not employ any quality measures or report on protocol violations.

\section{Statistical analysis}

We performed a network meta-analysis within a frequentist framework. We fit multivariate meta-analysis models with random effects using the network package in Stata, ${ }^{16,17}$ exploiting the direct and indirect randomized evidence to determine the relative effects and ranking. We reported the effects of the interventions using risk ratios (RRs) with 95\% confidence intervals (Cls). For the primary analysis on all emergency cesarean deliveries, we only included fetal surveillance types that were evaluated in more than 1 randomized trial, decided a priori to minimize the risk of inconsistency in the network. We also conducted a sensitivity analysis with all eligible trials reporting on the primary outcome, including those evaluated in only 1 trial. We calculated the mean rank and the surface under the cumulative ranking curve (SUCRA) for each intervention for all reported outcomes. Surveillance methods with a SUCRA value close to $100 \%$ had the highest cumulative rank (i.e., lowest likelihood) of achieving the adverse maternal or neonatal outcome..$^{18,19}$

We used the design-by-treatment interaction model to check the assumption of consistency in the entire network, assuming a 
common estimate for the heterogeneity variance across the different comparisons in the network. ${ }^{20}$ We investigated and detected inconsistency by comparing the direct and indirect evidence within the network using the node-splitting approach, ${ }^{21}$ assuming a common heterogeneity estimate within each loop (i.e., when numerous trials compare a minimum of 3 treatments to one another). ${ }^{22}$ We also investigated potential sources of inconsistency within relevant trials. In cases where inconsistency was detected, we conducted a network meta-regression compared with reference treatment (cardiotocography with fetal scalp blood $\mathrm{pH}$ sampling), testing for 2 potential effect modifiers, gestational age (preterm v. term) and pregnancy risk status (high risk v. low risk). ${ }^{23}$ All analyses were conducted using Stata statistical software, release 14 (StataCorp). ${ }^{16,19,24}$

\section{Results}

Of 351 potentially relevant citations, we included 33 randomized trials ( $n=120151$ patients) in our systematic review (Figure 1, Table 1). Most included trials were from high-income countries (United States, $n=6 ; ;^{26,27,32-34,38}$ United Kingdom, $n=5 ; 28,37,40,44,54$ Australia, $n=4 ; 3^{30,31,47,57}$ Sweden, $\left.n=4\right), 25,35,55,56$ except for 2 studies (Tanzania and India). ${ }^{41,42}$ The median sample size was 894 patients (range 100-47 062). Fifteen trials $(45 \%)^{25-27,31,32,36,39,41,43-46,49,51,53}$ included patients at 36 weeks' gestation or more, and the remaining included patients at 26-42 weeks' gestation. ${ }^{28-30,33-35,38,40,50,52,54,56}$ Two-thirds of trials (73\%) included patients with high-risk pregnancies, ${ }^{25-34,36,38,39,41,44,47,48,50,51,53,54} 3$ (9\%) included patients with lowrisk pregnancies $35,37,57$ and $4(12 \%)$ included pregnancies of any risk. ${ }^{40,42,43,52}$ Three studies did not report on pregnancy

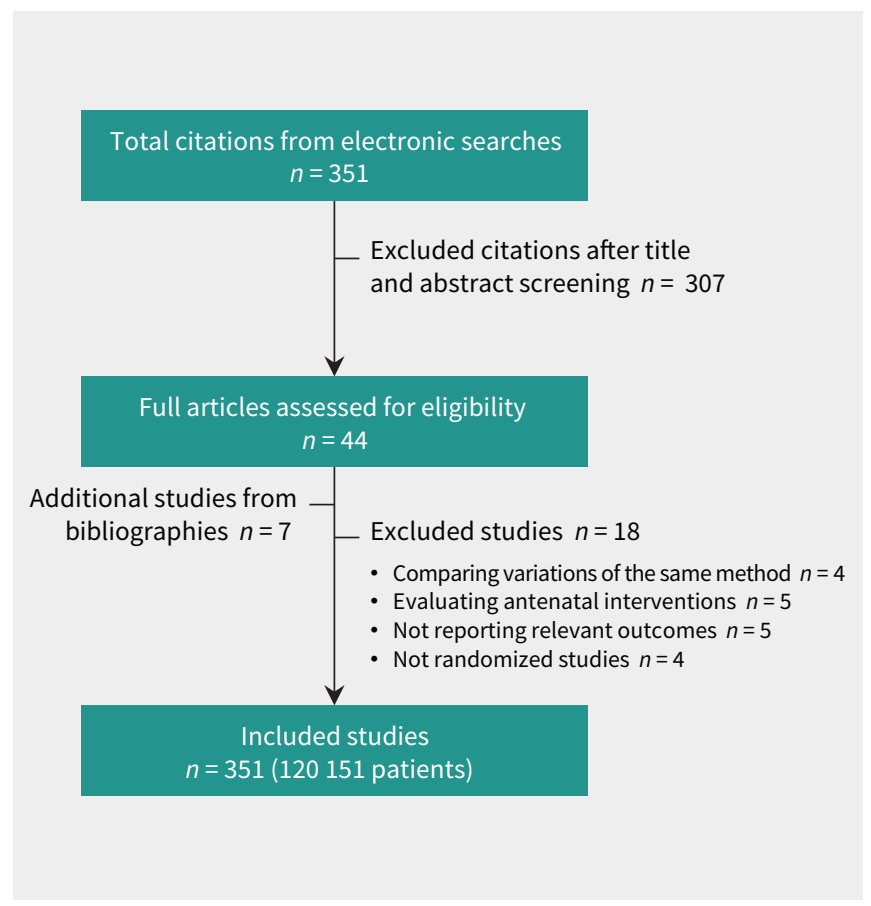

Figure 1: Flow chart of the study selection and inclusion process for network meta-analysis on intrapartum fetal surveillance. risks. ${ }^{45,46,49}$ The inclusion and exclusion criteria of each trial are summarized in Appendix 1, Supplementary Table 1.

Overall, we evaluated 10 fetal surveillance types, including intermittent auscultation (IA), cardiotocography (CTG) alone or in combination with computer-aided cardiotocography (cCTG), fetal scalp blood lactate sampling (CTG-lactate), fetal scalp blood $\mathrm{pH}$ sampling (CTG-FBS), fetal pulse oximetry (FPO-CTG), fetal heart electrocardiogram (STAN-CTG) and other combinations (STAN-CTG-FBS, FPO-CTG-FBS, CCTG-FBS).

All trials conducted head-to-head comparisons of 2 fetal surveillance methods with the exception of 1 trial that used a 3-arm design. ${ }^{34}$ Two methods, CCTG and STAN-CTG, were evaluated in only a single trial. ${ }^{26,36}$ These methods were therefore excluded from the primary analysis and were investigated in sensitivity analyses. Twenty-eight trials reported on all emergency cesarean deliveries in labour and 23 reported on cesarean deliveries for suspected fetal distress. Appendix 1 , Supplementary Table 3 summarizes the number of trials and participants contributing to each reported outcome per surveillance method. Appendix 1, Supplementary Table 4 summarizes the criteria that each trial used to interpret the findings of the fetal surveillance methods that it compared, as well as each trial's management algorithms and definitions of neonatal acidemia.

\section{Quality of the included studies}

The overall quality of included trials was moderate (Appendix 1, Supplementary Figure 1 and Supplementary Table 7). Trials that were conducted before the year 2000 showed high risk of bias in most of the evaluated domains. Ten trials (30\%) showed high risk of bias for randomization and 11 (33\%) for assignment concealment. Six trials showed high risk of bias for each of outcome assessment ${ }^{31,34,39,41,47,57}$ and selective reporting (18\%). ${ }^{31,33,39,41,47,57}$ Only 2 trials (6\%) had high loss to follow-up and data incompleteness. ${ }^{41,57}$ Overall, two-thirds of the included trials showed low risk of bias across the 5 evaluated elements.

\section{Emergency cesarean deliveries in labour}

Figure 2 illustrates the network of trials reporting on all emergency cesarean deliveries in labour (28 trials, including 103676 patients). Overall, IA reduced the risk of cesarean deliveries in labour compared with other methods (IA v. CTG: RR 0.83, 95\% Cl 0.72-0.97; IA v. CTG-FBS: RR 0.71, 95\% Cl 0.63-0.80; IA v. CTG-lactate: RR $0.77,95 \%$ Cl 0.64-0.92; IA v. FPO-CTG: RR 0.75, 95\% Cl 0.65-0.87; IA v. FPO-CTG-FBS: RR 0.81, 95\% Cl 0.67-0.99; cCTG-FBS v. IA: RR $1.21,95 \% \mathrm{Cl} 1.04-1.42)$ except when compared with STAN (STAN-CTG-FBS v. IA: RR 1.17, 95\% Cl 0.98-1.40) (Figure $3 \mathrm{~A}$ ). Intermittent auscultation was also most likely to reduce emergency cesarean deliveries (SUCRA 98.6), followed by STAN-CTG-FBS (SUCRA 72.3); CTG-FBS had the lowest likelihood of reducing the risk of emergency cesarean delivery (SUCRA 7.6) (Table 2, Figure 4). Using only CTG showed a similar effect on all emergency cesarean deliveries in labour compared with when it was combined with FBS (RR 1.17, 95\% Cl 0.99-1.40), STAN and FBS (RR $0.97,95 \% \mathrm{Cl} 0.89-1.07$ ) or with FPO (RR $1.02,95 \% \mathrm{Cl}$ 0.91-1.16) (Appendix 1, Supplementary Figure 2). We detected 
Table 1: Characteristics of included randomized trials on intrapartum fetal surveillance

\begin{tabular}{|c|c|c|c|c|c|c|}
\hline Study & Country & Settings & $\begin{array}{l}\text { Sample } \\
\text { size }\end{array}$ & Intervention & Comparison & $\begin{array}{c}\text { Gestation } \\
\text { age, wk }\end{array}$ \\
\hline Amer-Wahlin et al., $2001^{25}$ & Sweden & 3 labour wards & 4966 & STAN-CTG-FBS & CTG-FBS & $>36$ \\
\hline Belfort et al., $2015^{26}$ & US & 16 university maternity units & 11108 & STAN-CTG & CTG & $>36$ \\
\hline Bloom et al., $2006^{27}$ & US & 14 university maternity units & 5341 & CTG-FPO & CTG & $\geq 36$ \\
\hline Brocklehurst et al., $2017^{28}$ & UK & 24 maternity units & 47062 & cCTG-FBS & CTG-FBS & $\geq 35$ \\
\hline Caliskan et al., $2009^{29}$ & Turkey & Tertiary referral centre & 230 & CTG-FPO & CTG & $\geq 34$ \\
\hline East et al., $2006^{30}$ & Australia & 4 maternity hospitals & 601 & FPO-CTG-FBS & CTG-FBS & $\geq 26$ \\
\hline East et al., $2016^{31}$ & Australia & Tertiary referral centre & 123 & CTG-lactate & CTG & Full term \\
\hline Garite et al., $2000^{32}$ & US & 9 maternity hospitals & 1010 & FPO-CTG-FBS & CTG-FBS & $\geq 36$ \\
\hline Haverkamp et al., $1976^{33}$ & US & NR & 483 & CTG & IA & $\geq 35$ \\
\hline Haverkamp et al., $1979^{34}$ & US & General hospital & 690 & CTG & CTG-FBS & $>34$ \\
\hline Haverkamp et al., $1979^{34}$ & US & General hospital & 690 & CTG & IA & $>34$ \\
\hline Herbst et al., $1994^{35}$ & Sweden & Tertiary referral centre & 4044 & IA & CTG-FBS & $>33$ \\
\hline Ignatov et al., $2016^{36}$ & Bulgaria & Tertiary referral centre & 720 & cCTG & CTG-FBS & $\geq 37$ \\
\hline Kelso et al., $1978^{37}$ & UK & Tertiary referral centre & 504 & CTG & $\mathrm{IA}$ & NR \\
\hline Klauser et al., $2005^{38}$ & US & Hospital for women \& infants & 360 & CTG-FPO & CTG & $\geq 28$ \\
\hline Kuhnert et al., $2004^{39}$ & Germany & $\begin{array}{l}\text { Women's hospitals. (The German } \\
\text { Multicenter Study Group) }\end{array}$ & 146 & CTG-FBS-FPO & CTG-FBS & $\geq 36$ \\
\hline Macdonald et al., $1985^{40}$ & UK & Maternity hospital & 12964 & CTG-FBS & IA & $\geq 28$ \\
\hline Madaan et al., $2006^{41}$ & India & University hospital & 100 & CTG & IA & $\geq 37$ \\
\hline Mdoe et al., $2018^{42}$ & Tanzania & Referral hospital & 2652 & CTG & IA & NR \\
\hline Neldam et al., $1986^{43}$ & Denmark & $\begin{array}{l}\text { Labour ward in tertiary } \\
\text { university hospital }\end{array}$ & 1061 & CTG-FBS & IA & $\geq 36$ \\
\hline Nunes et al., $2017^{44}$ & UK & $\begin{array}{l}5 \text { hospitals, } 3 \text { tertiary care units } \\
\text { and } 2 \text { district general hospitals }\end{array}$ & 7730 & cCTG-FBS & CTG-FBS & $\geq 36$ \\
\hline Ojala et al., $2006^{45}$ & Finland & $\begin{array}{l}\text { Labour ward in tertiary } \\
\text { university hospital }\end{array}$ & 1483 & STAN-CTG-FBS & CTG-FBS & $\geq 36$ \\
\hline Puertas et al., $2018^{46}$ & Spain & University hospital & 237 & STAN-CTG-FBS & CTG-FBS & $\begin{array}{l}291-294 d \\
\text { gestation }\end{array}$ \\
\hline Renou et al., $1976^{47}$ & Australia & Fetal intensive care unit & 440 & CTG-FBS & IA & NR \\
\hline Strachan et al., $2000^{48}$ & $\begin{array}{l}\text { UK, Hong Kong, } \\
\text { Netherlands, } \\
\text { Singapore }\end{array}$ & 5 teaching hospitals & 1038 & STAN-CTG-FBS & CTG-FBS & NR \\
\hline Valverde et al., $2011^{49}$ & Spain & University hospital & 180 & STAN-CTG-FBS & FPO-CTG-FBS & Full term \\
\hline van Wijngaarden et al., $1996^{50}$ & UK, Hong Kong & 3 teaching hospitals & 214 & STAN-CTG-FBS & CTG-FBS & $>27$ \\
\hline Vayssiere et al., $2007^{51}$ & France & 2 maternity wards & 799 & STAN-CTG-FBS & CTG-FBS & $\geq 36$ \\
\hline Vintzileos et al., $1993^{52}$ & Greece & 2 university hospitals & 1428 & CTG & IA & $\geq 26$ \\
\hline Westerhuis et al., $2010^{53}$ & Netherlands & $\begin{array}{l}3 \text { academic and } 6 \text { nonacademic } \\
\text { teaching hospitals }\end{array}$ & 5681 & STAN-CTG-FBS & CTG-FBS & $>36$ \\
\hline Westgate et al. $1993^{54}$ & UK & Distric general hospital & 2434 & STAN-CTG-FBS & CTG-FBS & $>34$ \\
\hline Westgren et al. $1998^{55}$ & Sweden & University hospital & 341 & CTG-lactate & CTG-FBS & NR \\
\hline Wiberg-Itzel et al. $2008^{56}$ & Sweden & 10 labour ward departments & 2992 & CTG-lactate & CTG-FBS & $\geq 34$ \\
\hline Wood et al. $1981^{57}$ & Australia & $\begin{array}{l}\text { Maternity hospital and medical } \\
\text { centre }\end{array}$ & 989 & CTG-FBS & IA & NR \\
\hline
\end{tabular}

Note: $\mathrm{CCTG}=$ computer-aided cardiotocography, $\mathrm{cCTG}-\mathrm{FBS}=$ computer-aided cardiotocography with fetal scalp blood pH sampling, $\mathrm{CTG}=$ cardiotocography,

CTG-FBS = cardiotocography with fetal scalp blood pH sampling, CTG-lactate = cardiotocography with fetal scalp blood lactate, FPO-CTG = cardiotocography with fetal pulse oximetry, FPO-CTG-FBS = cardiotocography with fetal pulse oximetry and fetal blood pH sampling, IA = intermittent auscultation, NR = not reported, STAN-CTG = cardiotocograpy with fetal heart electrocardiogram, STAN-CTG-FBS = cardiotocography with fetal heart electrocardiogram and fetal scalp blood pH sampling. 


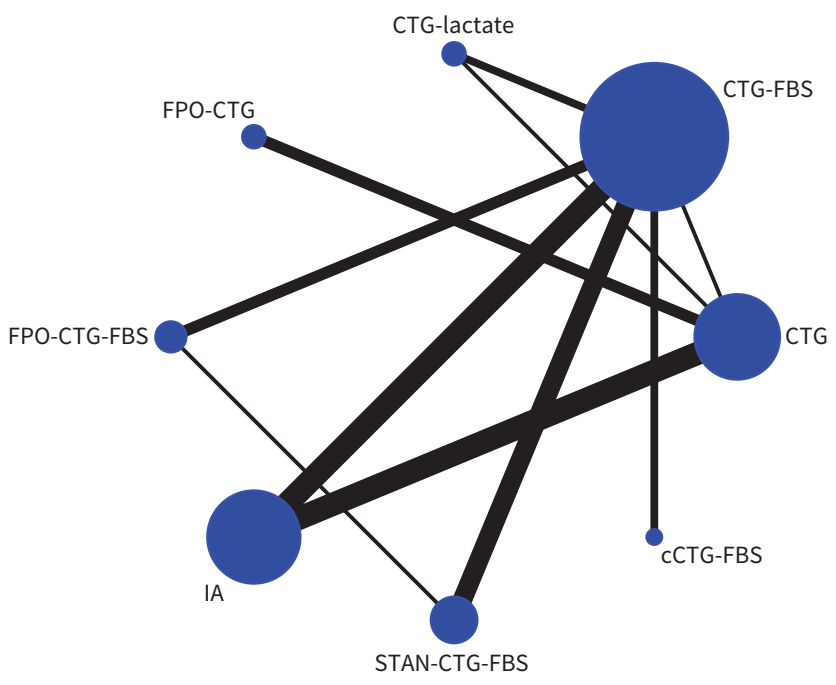

Figure 2: Network comparing the effectiveness of types of intrapartum fetal surveillance to reduce all emergency cesarean sections in labour. The dot size represents the number of participants in each comparison arm and the thickness of the lines represent the number of randomized trials directly comparing the pair of methods. Note: cCTG-FBS = computer-aided cardiotocography with fetal scalp blood $\mathrm{pH}$ sampling, $\mathrm{CTG}=$ cardiotocography, $\mathrm{CTG}-\mathrm{FBS}=$ cardiotocography with fetal scalp blood pH sampling, CTG-lactate = cardiotocography with fetal scalp blood lactate, FPO-CTG = cardiotocography with fetal pulse oximetry, FPO-CTG-FBS = cardiotocography with fetal pulse oximetry and fetal blood $\mathrm{pH}$ sampling, IA = intermittent auscultation, STAN-CTG-FBS = cardiotocography with fetal heart electrocardiogram and fetal scalp blood pH sampling.

significant inconsistency in this network $(p=0.01)$ that derived from evidence in the loop formed by CTG-FBS, FPO-CTG-FBS and STAN-CTG-FBS (Appendix 1, Supplementary Table 5).

Twenty-three trials reported on cesarean deliveries for suspected fetal distress ( $n=100500$ ) (Appendix 1, Supplementary Table 3). Intermittent auscultation showed a similar effect in reducing cesarean deliveries compared with CTG (RR 0.57, 95\% Cl 0.38-0.86), CTG-FBS (RR 0.34, $95 \% \mathrm{Cl} 0.22-0.50)$, FPO-CTG (RR $0.42,95 \% \mathrm{Cl} 0.25-0.70)$ and cCTG-FBS (RR 1.80, 95\% Cl 1.06-3.07) (Figure 3B). Combining FPO or STAN with CTG-FBS seemed to reduce the risk of cesarean deliveries for suspected fetal distress compared with using CTG-FBS alone (FPO-CTG-FBS v. CTG-FBS: RR 0.40, 95\% CI 0.21-0.77; STAN-CTG-FBS v. CTG-FBS: RR 0.47, $95 \% \mathrm{Cl} 0.26-0.84)$, though there was no significant difference between the 2 combinations when compared to one another (STAN-CTG-FBS v. FPO-CTG-FBS: RR 1.16, 95\% Cl 0.77-1.74) (Appendix 1, Supplementary Figure 3). There was significant inconsistency in this network $(p=0.004)$ that involved evidence loops for CTG-FBS, FPO-CTG-FBS and STAN-CTG-FBS (Appendix 1, Supplementary Table 5).

We further investigated the inconsistencies in the 12 trials that contributed to the evidence loop comparing CTG-FBS, FPO-CTG-FBS and STAN-CTG-FBS. Three trials compared FPOCTG-FBS to CTG-FBS, 8 trials compared STAN-CTG-FBS to CTGFBS and 1 trial compared STAN-CTG-FBS to FPO-CTG-FBS. All
12 trials were conducted in tertiary maternity units in highincome countries with similar study population characteristics. Two trials included patients with preterm pregnancies (CTGFBS-FPO v. CTG-FBS ${ }^{30}$ and CTG-FBS-STAN v. CTG-FBS ${ }^{50}$; t the others included patients at more than 36 weeks' gestation. Only 1 trial (CTG-FBS-FPO v. CTG-FBS ${ }^{39}$ ) showed an overall high risk of bias, with all remaining trials showing low to medium risk of bias. All trials followed established guidelines to interpret the findings of STAN and FPO in their action algorithms (Appendix 1, Supplementary Table 4).

We conducted a metaregression to investigate the potential effect of pregnancy status (high v. low risk) and gestational age (term v. preterm) on the risk for emergency cesarean deliveries and for cesarean deliveries for suspected fetal distress. Results suggested that pregnancy status had a significant effect among trials comparing FPO-CTG-FBS with CTG-FBS (Appendix 1, Supplementary Table 6). We conducted a sensitivity analysis, excluding trials evaluating FPO-CTG-FBS, which resolved the inconsistency in both networks without any major change in the effect sizes among remaining comparison arms (Appendix 1, Supplementary Figure 4).

\section{Secondary outcomes}

Using IA reduced the risk of all instrumental deliveries compared with using CTG alone (IA v. CTG: RR $0.82,95 \% \mathrm{Cl} 0.73-0.93$ ), but it did not show a significant difference compared with other fetal surveillance methods (Appendix 1, Supplementary Figure 5). In contrast, IA significantly reduced the risk of instrumental deliveries for suspected fetal distress compared with all other fetal surveillance methods (IA v. CTG: RR $0.41,95 \% \mathrm{Cl} 0.30-0.57$; IA v. CTG-FBS: RR 0.42, 95\% Cl 0.25-0.69; IA v. FPO-CTG-FBS: RR 0.50, 95\% Cl 0.32-0.79; STAN-CTG-FBS v. IA: RR 2.14, 95\% Cl 1.49-3.08; cCTG-FBS v. IA: RR 2.52, 95\%Cl 1.77-3.60) (Appendix 1, Supplementary Figure 6).

All types of fetal surveillance had a similar effect on the evaluated neonatal outcomes (neonatal acidemia, admission to neonatal unit, Apgar $<7$ at 5th minute and perinatal death) (Appendix 1, Supplementary Figure 7-10). Table 2 shows the ranking of all evaluated types of surveillance according to their likelihood of reducing adverse neonatal outcomes. Combining FPO or STAN with CTG-FBS seemed to improve the likelihood of reducing adverse neonatal outcomes, but this did not result in a significant difference in our network meta-analysis. We did not detect significant inconsistency in the evidence networks for any of the secondary outcomes.

\section{Sensitivity analysis}

Our network meta-analysis that included the 2 trials evaluating the use of STAN-CTG and CCTG alone on emergency cesarean deliveries showed that the use of STAN-CTG had a similar effect on the risk of all emergency cesarean deliveries compared with other surveillance methods except STAN-CTG-FBS (RR 0.79, 95\% Cl 0.64-0.98) and IA (RR 1.47, 95\% Cl 1.28-1.70) (Appendix 1, Supplementary Figures 11 and 12). A similar effect was seen for cesarean deliveries for suspected fetal distress (Appendix 1, Supplementary Figure 12). 
A

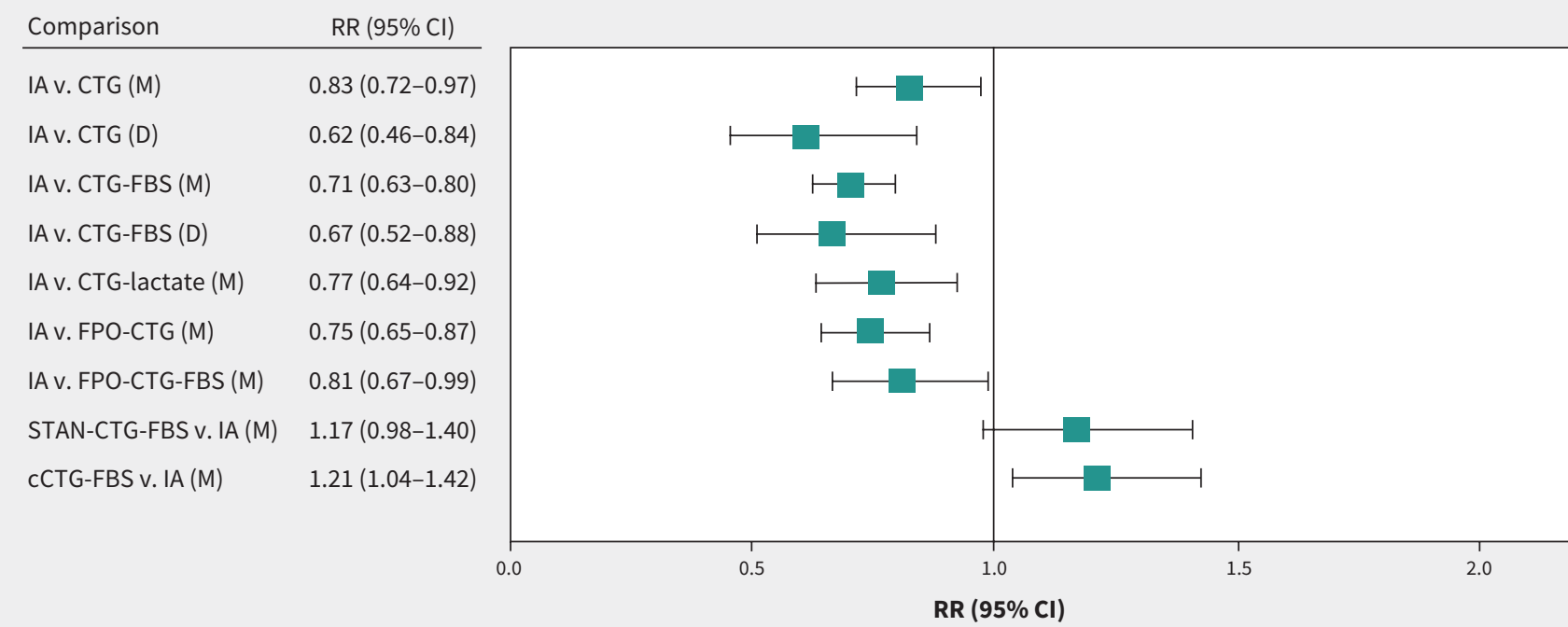

B

\begin{tabular}{ll} 
Comparison & RR $(95 \% \mathrm{CI})$ \\
\hline IA v. CTG (M) & $0.57(0.38-0.86)$ \\
IA v. CTG (D) & $0.30(0.13-0.67)$ \\
IA v. CTG-FBS (M) & $0.34(0.22-0.50)$ \\
IA v. CTG-FBS (D) & $0.57(0.37-0.88)$ \\
IA v. FPO-CTG (M) & $0.42(0.25-0.70)$ \\
IA v. FPO-CTG-FBS (M) & $0.83(0.47-1.45)$ \\
STAN-CTG v. IA (M) & $1.39(0.86-2.26)$ \\
CCTG-FBS v. IA (M) & $1.80(1.06-3.07)$
\end{tabular}

Figure 3: Network meta-analysis comparing the effectiveness of intermittent auscultation (IA) to reduce (A) all emergency cesarean deliveries in labour and (B) emergency cesarean deliveries for suspected fetal distress. The forest plots depict the effectiveness of IA compared with other types of fetal surveillance using both mixed evidence network meta-analysis (M) and direct evidence pairwise meta-analysis (D). Note: cCTG-FBS = computer-aided cardiotocography with fetal scalp blood pH sampling, CTG = cardiotocography, CTG-FBS = cardiotocography with fetal scalp blood pH sampling, CTGlactate $=$ cardiotocography with fetal scalp blood lactate, FPO-CTG $=$ cardiotocography with fetal pulse oximetry, FPO-CTG-FBS $=$ cardiotocography with fetal pulse oximetry and fetal blood pH sampling, $\mathrm{LCL}=$ lower confidence limit, $\mathrm{RR}=$ risk ratio, STAN-CTG-FBS = cardiotocography with fetal heart electrocardiogram and fetal scalp blood pH sampling, $\mathrm{UCL}=$ upper confidence limit.

Network meta-analysis on the use of CCTG alone suggested a consistent reduction in the relative risk for all emergency cesarean deliveries by around 50\% compared with all other surveillance methods (Appendix 1, Supplementary Figure 11). No data were available on the effect of CCTG on cesarean deliveries for suspected fetal distress. Given the potential high risk of bias and inconsistency between the indirect and the direct evidence sought from this small trial, we interpret these findings with caution.

\section{Interpretation}

Despite the large investment in developing intrapartum fetal surveillance technology over the last 5 decades, our network metaanalysis suggests that none of the newer surveillance methods seem to significantly reduce emergency cesarean deliveries in labour or neonatal morbidity compared with using simple IA of the fetal heart. Combining newer methods such as STAN and FPO with CTG may improve its performance, thus reducing 
Table 2: Summary of the calculated surface under the cumulative ranking curve (SUCRA) and mean rank for types of

intrapartum fetal surveillance across maternal and neonatal outcomes*

\begin{tabular}{|c|c|c|c|c|c|c|c|c|c|c|c|c|c|c|c|c|}
\hline \multirow[b]{2}{*}{ Outcomes } & \multicolumn{2}{|c|}{ IA } & \multicolumn{2}{|c|}{ CTG } & \multicolumn{2}{|c|}{ CTG-FBS } & \multicolumn{2}{|c|}{ CTG-lactate } & \multicolumn{2}{|c|}{ STAN-CTG-FBS } & \multicolumn{2}{|c|}{ FPO-CTG } & \multicolumn{2}{|c|}{ FPO-CTG-FBS } & \multicolumn{2}{|c|}{ cCTG-FBS } \\
\hline & $\begin{array}{c}\text { SUCRA } \\
\%\end{array}$ & $\begin{array}{l}\text { Mean } \\
\text { rank }\end{array}$ & $\begin{array}{c}\text { SUCRA } \\
\%\end{array}$ & $\begin{array}{l}\text { Mean } \\
\text { rank }\end{array}$ & $\begin{array}{c}\text { SUCRA } \\
\%\end{array}$ & $\begin{array}{l}\text { Mean } \\
\text { rank }\end{array}$ & $\begin{array}{c}\text { SUCRA } \\
\%\end{array}$ & $\begin{array}{l}\text { Mean } \\
\text { rank }\end{array}$ & $\begin{array}{c}\text { SUCRA } \\
\%\end{array}$ & $\begin{array}{l}\text { Mean } \\
\text { rank }\end{array}$ & $\begin{array}{c}\text { SUCRA } \\
\%\end{array}$ & $\begin{array}{l}\text { Mean } \\
\text { rank }\end{array}$ & $\begin{array}{c}\text { SUCRA } \\
\%\end{array}$ & $\begin{array}{l}\text { Mean } \\
\text { rank }\end{array}$ & $\begin{array}{c}\text { SUCRA } \\
\%\end{array}$ & $\begin{array}{l}\text { Mean } \\
\text { rank }\end{array}$ \\
\hline \multicolumn{17}{|c|}{ Maternal outcomes } \\
\hline Any EmCD & 98.6 & 1.1 & 63.1 & 3.6 & 7.6 & 7.5 & 25.4 & 6.2 & 72.3 & 2.9 & 30.8 & 5.8 & 49.0 & 4.6 & 53.2 & 4.3 \\
\hline $\begin{array}{l}\text { Any } \\
\text { Instrumental } \\
\text { delivery }\end{array}$ & 87.6 & 1.9 & 25.7 & 6.2 & 64.6 & 3.5 & 45.6 & 4.8 & 22.3 & 6.4 & 63.0 & 3.6 & 36.5 & 5.4 & $\mathrm{~N} / \mathrm{A}$ & N/A \\
\hline $\begin{array}{l}\text { Instrumental } \\
\text { delivery for } \\
\text { suspected } \\
\text { fetal distress }\end{array}$ & 100.0 & 1.0 & 26.3 & 4.7 & 33.5 & 4.3 & N/A & $\mathrm{N} / \mathrm{A}$ & 57.7 & 3.1 & $\mathrm{~N} / \mathrm{A}$ & $\mathrm{N} / \mathrm{A}$ & 64.7 & 2.8 & 17.8 & 5.1 \\
\hline Acidemia & 51.8 & 4.4 & 38.9 & 5.3 & 15.5 & 6.9 & 63.7 & 3.5 & 71.2 & 3.0 & 35.5 & 5.5 & 76.2 & 2.7 & 47.2 & 4.7 \\
\hline $\begin{array}{l}\text { Admission to } \\
\text { NICU }\end{array}$ & 42.1 & 5.1 & 38.3 & 5.3 & 49.3 & 4.6 & 37.0 & 5.4 & 75.7 & 2.7 & 78.3 & 2.5 & 10.5 & 7.3 & 68.9 & 3.2 \\
\hline $\begin{array}{l}\text { Apgar }<7 \text { at } \\
5 \text { th minute }\end{array}$ & 75.9 & 2.7 & 26.3 & 6.2 & 64.4 & 3.5 & 13.9 & 7.0 & 39.2 & 5.3 & 52.4 & 4.3 & 79.4 & 2.4 & 48.4 & 4.6 \\
\hline $\begin{array}{l}\text { Perinatal } \\
\text { death }\end{array}$ & 44.8 & 4.3 & 46.8 & 4.2 & 69.1 & 2.9 & $\mathrm{~N} / \mathrm{A}$ & $\mathrm{N} / \mathrm{A}$ & 36.0 & 4.8 & 80.0 & 2.2 & 32.1 & 5.1 & 41.2 & 4.5 \\
\hline
\end{tabular}

Note: $\mathrm{cCTG}-\mathrm{FBS}=$ computer-aided cardiotocography with fetal scalp blood pH sampling, CTG = cardiotocography, CTG-FBS = cardiotocography with fetal scalp blood pH sampling, CTG-lactate = cardiotocography with fetal scalp blood lactate, EmCD = emergency cesarean delivery, FPO-CTG = cardiotocography with fetal pulse oximetry, FPO-CTG-FBS = cardiotocography with fetal pulse oximetry and fetal blood pH sampling, IA = intermittent auscultation, N/A = no available evidence, NICU = neonatal intensive care unit, STAN-CTGFBS = cardiotocography with fetal heart electrocardiogram and fetal scalp blood pH sampling.

*The calculated SUCRA for all adverse outcomes expresses the lowest likelihood of developing adverse maternal and neonatal outcomes.

unnecessary cesarean deliveries. However, none of these combinations were shown to significantly outperform IA in reducing adverse maternal and neonatal outcomes. Although there were variations in the quality and reporting across trials, leading to inconsistency in the evidence networks on cesarean delivery outcomes, the effect estimates favouring IA across all evaluated types of surveillance were consistent in our sensitivity analyses, thereby increasing the generalizability of our findings. Trials evaluating the use of FPO contributed to statistical inconsistency when evaluating the incidence of emergency cesarean deliveries. As such, the direct and indirect evidence on the effect of FPO should be interpreted with caution, pending larger trials to better evaluate its effectiveness.

Our review provides a uniquely comprehensive evidence synthesis on all current types of fetal surveillance for intrapartum care. We leveraged both direct and indirect evidence from randomized trials using the network meta-analysis methodology to compare and rank types of surveillance not previously evaluated in pairwise meta-analyses (e.g., IA v. STAN). Our systematic review adopted a pragmatic approach to report on all relevant endpoints, as reported by the authors, to synthesize the most comprehensive evidence on the topic that could directly apply to clinical practice. We assessed the risk of bias using a standard tool and took into account any quality improvement measures to improve the validity of each trial, given the complexity of the evaluated interventions and the chance of methodological heterogeneity. To reduce the risk of inconsistency in our network, we eliminated methods of surveillance evaluated by a single trial (CCTG and CTG-STAN) from the primary analysis and performed supplementary analyses to assess the true effect of these methods. We examined for inconsistency using the node-split approach and conducted a meta-regression, which found that the detected inconsistency could be explained by whether pregnancy status was high or low risk.

All currently available methods of intrapartum fetal surveillance assume that detecting subtle changes and variability in the fetal heart rate could identify fetuses at risk of intrapartum asphyxia, prompting further interventions. ${ }^{58}$ Simultaneously, fetal surveillance aims to identify those fetuses coping well with labour, regardless of the subtle changes in the fetal heart rate, to avoid unnecessary interventions, reassure mothers and their health care providers. ${ }^{58}$ Introducing IA into practice helped to improve the detection of acutely compromised fetuses after serious events such as placental abruption, thus reducing associated adverse outcomes. ${ }^{59}$ However, its accuracy to detect subacute hypoxia and chronic intrapartum insult remains debatable. ${ }^{3}$ Our 


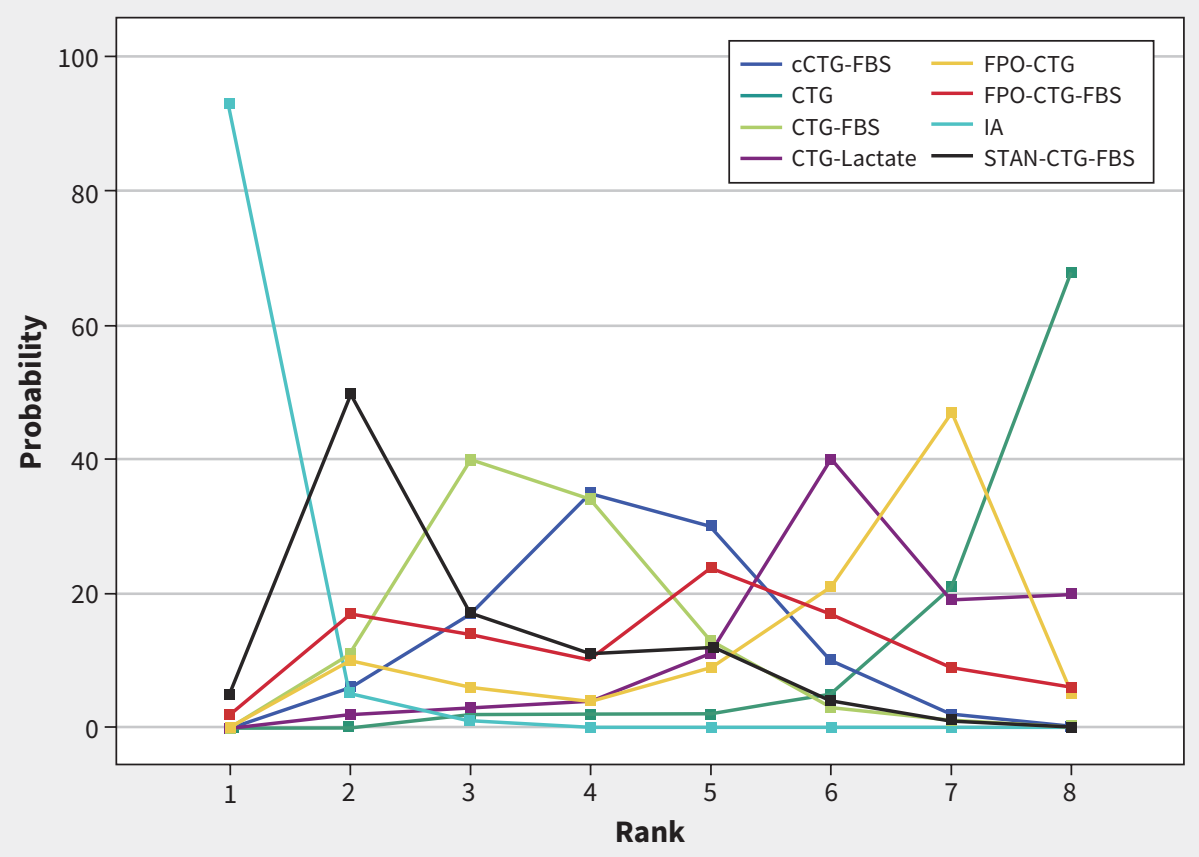

Figure 4: The ranking probability of types of intrapartum fetal surveillance to reduce all emergency cesarean deliveries when used in labour. The graph depicts the probability of each type to be ranked the highest in reducing the incidence of all emergency cesarean deliveries in labour. IA was consistently ranked first, followed by STAN-CTG-FBS and CTG-FBS. Note: CCTG = computer-aided cardiotocography, cCTG-FBS = computer-aided cardiotocography with fetal scalp blood pH sampling, CTG = cardiotocography, CTG-FBS = cardiotocography with fetal scalp blood pH sampling, CTG-lactate = cardiotocography with fetal scalp blood lactate, FPO-CTG = cardiotocography with fetal pulse oximetry, FPO-CTG-FBS = cardiotocography with fetal pulse oximetry and fetal blood pH sampling, IA = intermittent auscultation, STAN-CTG = cardiotocography with fetal heart electrocardiogram, STANCTG-FBS = cardiotocography with fetal heart electrocardiogram and fetal scalp blood pH sampling.

analysis suggests that all additional methods introduced to improve the accuracy of electronic fetal heart monitoring (CTG, FBS, FPO, STAN and CCTG) have failed to reduce the risk of adverse neonatal or maternal outcomes beyond what IA achieved 50 years ago, and this may have contributed to the increased incidence of unnecessary emergency cesarean deliveries. As such, the benefit of these newer surveillance methods is questionable, especially in view of the consistent rise in the rate of cesarean deliveries worldwide. The increasing incidence of cesarean deliveries is particularly notable in high-income countries where advanced fetal surveillance methods are readily available. The suboptimal performance of newer fetal surveillance methods could be mediated by a higher false-positive detection rate or human errors in interpreting their outputs, driven by operator incompetence or increased interface complexity. Clearly, the rising rate of cesarean deliveries is multifactorial ${ }^{60}$ and cannot be linked solely to poorly performing intrapartum fetal surveillance methods. More specifically, our findings suggest that these technologies failed to meet their objective of reducing unnecessary interventions in labour.

Most included trials failed to report on key long-term neonatal outcomes, such as cerebral palsy, neonatal seizures and hypoxic-ischemic encephalopathy. Instead, most reported on short-term, readily recorded outcomes, such as neonatal acidemia and Apgar scores, all of which have a modest correlation with long-term child development. ${ }^{61}$ Similarly, there was poor reporting on important long-term maternal outcomes following cesarean deliveries and instrumental deliveries, such as subsequent placenta accreta and anal sphincter injuries. ${ }^{62}$ Those long-term outcomes should be considered in future trials and their follow-ups, especially given the contrast between randomized and observational evidence on the effect of adopting newer fetal surveillance methods in practice. ${ }^{4}$

Given the inconsistency in evidence on the use of FPO and STAN compared with CTG-FBS, large, randomized trials are needed to determine their effectiveness in both high- and lowrisk pregnancies when implemented in routine practice, especially given the potential high costs (e.g., hardware, software and staff training). Novel study designs such as multiarm, multistage comparison and cluster randomization trials may be better suited to evaluate the efficacy and effectiveness of fetal surveillance in everyday clinical settings. Specifically, these trials should aim to evaluate the preferential use of IA and CTG in lowv. high-risk pregnant people as well as the effectiveness of different fetal surveillance algorithms across different health care settings (e.g high- v. low-income countries).

Developing and evaluating new fetal surveillance methods is a complex, expensive and time-consuming endeavour because of 
the increased safety requirements in clinical research. Given the modest improvement in neonatal and maternal outcomes over the last 50 years, boosting collaboration among researchers, funders, industry, policymakers and lay consumers is required to tackle this important health issue. ${ }^{63}$ The rapid improvement in communication technology could facilitate this objective and foster transcountry collaborations to aid joint research efforts and data sharing. Our meta-analysis makes the case for serious investment in the development and evaluation of novel intrapartum surveillance technologies to make birth a safer process for patients and their offspring.

\section{Limitations}

Several of the trials included in our meta-analysis were relatively old. Their quality differs compared with newer trials, especially for the elements of randomization, assignment concealment and outcome assessment. This was particularly relevant to trials comparing IA and CTG. ${ }^{33-35,37,40-43,47,52,57}$ Limited reporting on attrition, and the use of intention-to-treat analysis were provided in some trials, particularly those before 2000 . We included these trials to minimize research waste, but also to highlight the elements of suboptimal design. Furthermore, we assessed their risk of bias to offer a comprehensive overview of changes in fetal surveillance over time. Several older trials included participants with different pregnancy status (high v. low risk) using different selection criteria. This could have an impact on effect sizes, though we did not detect a statistically significant effect in our meta-regression. A number of trials adopted different criteria for interpreting CTGs and fetal wellbeing, which might bias the findings. However, none of the evaluated criteria seemed to offer better accuracy for detecting fetal compromise in labour. ${ }^{64}$ The definition of neonatal acidemia has evolved over the years, which resulted in variation across trials. We were unable to accommodate several potential effect modifiers, such as progress in labour, induction of labour, suspected fetal growth restriction, use of oxytocin augmentation, operator experience and deviation from the trial's protocol. Although some of these elements could be adjusted for in an individual patient data meta-analysis, several factors are difficult to measure in practice, such as severity of sepsis in labour and degree of fetal growth restriction. Lastly, most of the included trials were underpowered to detect rare adverse neonatal outcomes and were potentially affected by the Hawthorne effect, limiting their generalizability. Our comprehensive analysis therefore offers a pragmatic approach that synthesizes the best quality evidence available to-date and guides future research efforts to address these limitations.

\section{Conclusion}

Compared with other types of fetal surveillance, intermittent auscultation seems to reduce emergency cesarean deliveries in labour without increasing adverse neonatal and maternal outcomes. New fetal surveillance methods did not improve neonatal outcomes or reduce unnecessary maternal interventions. Further evidence is needed to evaluate the effects of fetal pulse oximetry and fetal heart electrocardiography in labour.

\section{References}

1. Jibodu OA, Arulkumaran S. Intrapartum fetal surveillance. Curr Opin Obstet Gynecol 2000;12:123-7.

2. Intrapartum care for healthy women and babies. London (UK): The National Institute for Health and Care Excellence; 2014; Available: https://www.nice. org.uk/guidance/cg190 (accessed 2021 Jan. 1).

3. Steer PJ. Has electronic fetal heart rate monitoring made a difference? Semin Fetal Neonat Med 2008;13:2-7.

4. Olofsson P, Ayres de Campos D, Kessler J, et al. A critical appraisal of the evidence for using cardiotocography plus ECG ST interval analysis for fetal surveillance in labor. Part II: the meta analyses. Acta Obstet Gynecol Scand 2014;93:571-86.

5. Carbonne B, Pons K, Maisonneuve E. Foetal scalp blood sampling during labour for pH and lactate measurements. Best Pract Res Clin Obstet Gynaecol 2016;30:62-7.

6. INFANT Collaborative Group. Computerised interpretation of fetal heart rate during labour (INFANT): a randomised controlled trial. Lancet 2017;389:1719-29.

7. Molina G, Weiser TG, Lipsitz SR, et al. Relationship between cesarean delivery rate and maternal and neonatal mortality. JAMA 2015;314:2263-70.

8. Blencowe $\mathrm{H}$, Cousens $\mathrm{S}$, Jassir FB, et al. National, regional, and worldwide estimates of stillbirth rates in 2015, with trends from 2000: a systematic analysis. Lancet Glob Health 2016;4:e98-108.

9. Thacker SB, Stroup D, Chang M. Continuous electronic heart rate monitoring for fetal assessment during labor. Cochrane Database Syst Rev 2001;(2):CD000063.

10. Alfirevic Z, Gyte GML, Cuthbert A, et al. Continuous cardiotocography (CTG) as a form of electronic fetal monitoring (EFM) for fetal assessment during labour. Cochrane Database Syst Rev 2017;(2):CD006066.

11. East CE, Begg L, Colditz PB, et al. Fetal pulse oximetry for fetal assessment in labour. Cochrane Database Syst Rev 2014;(10):CD004075.

12. Martis R, Emilia O, Nurdiati DS, et al. Intermittent auscultation (IA) of fetal heart rate in labour for fetal well being. Cochrane Database Syst Rev 2017;(2): CD008680.

13. Saccone G, Schuit E, Am-Wåhlin I, et al. Electrocardiogram ST analysis during labor: a systematic review and meta-analysis of randomized controlled trials. Obstet Gynecol 2016;127:127-35.

14. Hutton B, Salanti G, Caldwell DM, et al. The PRISMA extension statement for reporting of systematic reviews incorporating network meta-analyses of health care interventions: checklist and explanations. Ann Intern Med 2015;162:777-84.

15. Higgins JPT, Altman DG, Gøtzsche PC, et al. The Cochrane Collaboration's tool for assessing risk of bias in randomised trials. BMJ 2011;343:d5928.

16. White IR, Barrett JK, Jackson D, et al. Consistency and inconsistency in net work meta analysis: model estimation using multivariate meta regression. Res Synth Methods 2012;3:111-25.

17. White IR. Network meta-analysis. Stata J 2015;15:951-85

18. Salanti G, Ades AE, loannidis JPA. Graphical methods and numerical summaries for presenting results from multiple-treatment meta-analysis: an overview and tutorial. J Clin Epidemiol 2011;64:163-71.

19. Chaimani A, Higgins JPT, Mavridis D, et al. Graphical tools for network metaanalysis in STATA. PLoS One 2013;8:e76654.

20. Higgins JPT, Jackson D, Barrett JK, et al. Consistency and inconsistency in network meta analysis: concepts and models for multiarm studies. Res Synth Methods 2012;3:98-110.

21. Dias S, Welton NJ, Caldwell DM, et al. Checking consistency in mixed treatment comparison meta analysis. Stat Med 2010;29:932-44

22. Veroniki AA, Vasiliadis HS, Higgins JPT, et al. Evaluation of inconsistency in networks of interventions. Int J Epidemiol 2013;42:332-45.

23. Dias S, Ades AE, Welton NJ, et al. Network meta-analysis for decision-making. Hoboken (NJ): John Wiley \& Sons; 2018.

24. White IR. Multivariate random-effects meta-regression: updates to mvmeta. Stata J 2011;11:255-70.

25. Amer-Wåhlin I, Hellsten C, Norén H, et al. Cardiotocography only versus cardiotocography plus ST analysis of fetal electrocardiogram for intrapartum fetal monitoring: a Swedish randomised controlled trial. Lancet 2001;358:534-8.

26. Belfort MA, Saade GR, Thom E, et al. A randomized trial of intrapartum fetal ECG ST-segment analysis. N Engl J Med 2015;373:632-41.

27. Bloom SL, Spong CY, Thom E, et al. Fetal pulse oximetry and cesarean delivery. N Engl J Med 2006;355:2195-202.

28. INFANT Collaborative Group. Computerised interpretation of fetal heart rate during labour (INFANT): a randomised controlled trial. Lancet 2017;389:1719-29.

29. Caliskan E, Cakiroglu Y, Corakci A, et al. Reduction in caesarean delivery with fetal heart rate monitoring and intermittent pulse oximetry after induction of labour with misoprostol. J Matern Fetal Neonatal Med 2009;22:445-51.

30. East CE, Brennecke SP, King JF, et al., FOREMOST Study Group. The effect of intrapartum fetal pulse oximetry, in the presence of a nonreassuring fetal heart rate pattern, on operative delivery rates: a multicenter, randomized, controlled trial (the FOREMOST trial). Am J Obstet Gynecol 2006;194:606-e1-16. 
31. East C, Davey MA, Kamlin O, et al. Randomised trial of fetal scalp blood sampling for lactate measurement: The Flamingo trial. BJOG 2016;123.

32. Garite TJ, Dildy GA, McNamara H, et al. A multicenter controlled trial of fetal pulse oximetry in the intrapartum management of nonreassuring fetal heart rate patterns. Am J Obstet Gynecol 2000;183:1049-58.

33. Haverkamp AD, Thompson HE, McFee JG, et al. The evaluation of continuous fetal heart rate monitoring in high-risk pregnancy. Am J Obstet Gynecol 1976; 125:310-20.

34. Haverkamp AD, Orleans M, Langendoerfer S, et al. A controlled trial of the differential effects of intrapartum fetal monitoring. Am J Obstet Gynecol 1979;134:399-412.

35. Herbst A, Ingemarsson I. Intermittent versus continuous electronic monitoring in labour: a randomised study. Br J Obstet Gynaecol 1994;101:663-8.

36. Ignatov PN, Lutomski JE. Quantitative cardiotocography to improve fetal assessment during labor: a preliminary randomized controlled trial. Eur J Obstet Gynecol Reprod Biol 2016;205:91-7.

37. Kelso IM, Parsons RJ, Lawrence GF, et al. An assessment of continuous fetal heart rate monitoring in labor: a randomized trial. Am J Obstet Gynecol 1978;131:526-32.

38. Klauser CK, Christensen EE, Chauhan SP, et al. Use of fetal pulse oximetry among high-risk women in labor: a randomized clinical trial. Am J Obstet Gynecol 2005;192:1810-7.

39. Kühnert M, Schmidt S. Intrapartum management of nonreassuring fetal heart rate patterns: a randomized controlled trial of fetal pulse oximetry. Am J Obstet Gynecol 2004;192:1989-95.

40. MacDonald D, Grant A, Sheridan-Pereira M, et al. The Dublin randomized controlled trial of intrapartum fetal heart rate monitoring. Am J Obstet Gynecol 1985;152:524-39.

41. Madaan M, Trivedi SS. Intrapartum electronic fetal monitoring vs. intermittent auscultation in postcesarean pregnancies. Int J Gynaecol Obstet 2006;94:123-5.

42. Mdoe PF, Ersdal HL, Mduma E, et al. Randomized controlled trial of continuous Doppler versus intermittent fetoscope fetal heart rate monitoring in a low resource setting. Int J Gynaecol Obstet 2018;143:344-50.

43. Neldam S, Osler M, Hansen PK, et al. Intrapartum fetal heart rate monitoring in a combined low-and high-risk population: a controlled clinical trial. Eur J Obstet Gynecol Reprod Biol 1986;23:1-11.

44. Nunes I, Ayres-de-Campos D, Ugwumadu A, et al. Central fetal monitoring with and without computer analysis. Obstet Gynecol 2017;129:83-90.

45. Ojala K, Vääräsmäki M, Mäkikallio K, et al. A comparison of intrapartum automated fetal electrocardiography and conventional cardiotocography-a randomised controlled study. BJOG 2006;113:419-23.

46. Puertas A, Góngora J, Valverde M, et al. Cardiotocography alone vs. cardiotocography with ST segment analysis for intrapartum fetal monitoring in women with late-term pregnancy. A randomized controlled trial. Eur J Obstet Gynecol Reprod Biol 2019;234:213-7.
47. Renou P, Chang A, Anderson I, et al. Controlled trial of fetal intensive care. Am J Obstet Gynecol 1976;126:470-6.

48. Strachan BK, van Wijngaarden WJ, Sahota D, et al., FECG Study Group. Cardiotocography only versus cardiotocography plus PR-interval analysis in intrapartum surveillance: a randomised, multicentre trial. Lancet 2000;355:456-9.

49. Valverde M, Puertas AM, Lopez-Gallego MF, et al. Effectiveness of pulse oximetry versus fetal electrocardiography for the intrapartum evaluation of nonreassuring fetal heart rate. Eur J Obstet Gynecol Reprod Biol 2011;159:333-7.

50. van Wijngaarden WJ, Sahota DS, James DK, et al. Improved intrapartum surveillance with PR interval analysis of the fetal electrocardiogram: a randomized trial showing a reduction in fetal blood sampling. Am J Obstet Gynecol 1996;174:1295-9.

51. Vayssière C, David E, Meyer N, et al. A French randomized controlled trial of STsegment analysis in a population with abnormal cardiotocograms during labor. Am J Obstet Gynecol 2007;197:299-e1.

52. Vintzileos AM, Antsaklis A, Varvarigos I, et al. A randomized trial of intrapartum electronic fetal heart rate monitoring versus intermittent auscultation. Obstet Gynecol 1993;81:899-907.

53. Westerhuis ME, Moons KG, van Beek E, et al. A randomised clinical trial on cardiotocography plus fetal blood sampling versus cardiotocography plus STanalysis of the fetal electrocardiogram (STAN $\left.{ }^{\circledR}\right)$ for intrapartum monitoring. BMC Pregnancy Childbirth 2007;7:1-8.

54. Westgate J, Harris M, Curnow JS, et al. Randomised trial of cardiotocography alone or with ST waveform analysis for intrapartum monitoring. Lancet 1992;340:194-8.

55. Westgren M, Kruger K, Ek S, et al. Lactate compared with $\mathrm{pH}$ analysis at fetal scalp blood sampling: a prospective randomised study. BJOG 1998;105:29-33.

56. Wiberg-Itzel E, Lipponer C, Norman M, et al. Determination of pH or lactate in fetal scalp blood in management of intrapartum fetal distress: randomised controlled multicentre trial. BMJ 2008;336:1284-7.

57. Wood C, Renou P, Oats J, et al. A controlled trial of fetal heart rate monitoring in a low-risk obstetric population. Am J Obstet Gynecol 1981;141:527-34.

58. Devoe LD. Future perspectives in intrapartum fetal surveillance. Best Pract Res Clin Obstet Gynaecol 2016;30:98-106.

59. Goddard R. Electronic fetal monitoring: is not necessary for low risk labours BMJ 2001;322:1436-7.

60. Betrán AP, Torloni MR, Zhang J-J, et al. WHO statement on caesarean section rates. BJOG 2016;123:667-70.

61. Sabol BA, Caughey AB. Acidaemia in neonates with a 5-minute Apgar score of 7 or greater - What are the outcomes? Am J Obstet Gynecol 2016;215:486.e1-6.

62. Grivell RM, Dodd JM. Short-and long-term outcomes after cesarean section. Expert Rev of Obstet Gynecol 2011;6:205-15.

63. Adam J. The future of fetal monitoring. Rev Obstet Gynecol 2012;5:e132-6.

64. Garabedian C, Butruille L, Drumez E, et al. Inter-observer reliability of 4 fetal heart rate classifications. J Gynecol Obstet Hum Reprod 2017;46:131-5.

\section{Competing interests: None declared.}

This article has been peer reviewed.

Affiliations: Warwick Medical School (Al Wattar, Honess, Bunnewell, Quenby), University of Warwick, Coventry, UK; Reproductive Medicine Unit (Al Wattar), University College London Hospitals, London, UK; Population Health Sciences (Welton), Bristol Medical School, University of Bristol, Bristol, UK; University Hospital of Coventry and Warwickshire (Quenby), Coventry, UK; Department of Preventive Medicine and Public Health (Khan), University of Granada, Granada, Spain; Clinical Biostatistics Unit, Ramon y Cajal Hospital (IRYCIS) and CIBER Epidemiology and Public Health (Khan, Zamora), Madrid, Spain; CIBER of Epidemiology and Public Health (CIBERESP) (Zamora), Madrid, Spain; WHO Collaborating Centre for Global Women's Health, Institute of Metabolism and Systems Research (Thangaratinam), University of Birmingham; Birmingham Women's and Children's NHS Foundation Trust (Thangaratinam), Birmingham, UK
Contributors: Bassel Al Wattar conceived and designed the study. Bassel Al Wattar, Emma Honess and Sarah Bunnewell acquired the data. All authors contributed to the analysis and interpretation of data. Bassel Al Wattar drafted the initial manuscript, which all authors critically revised for important intellectual content. All authors gave final approval of the version to be published and agreed to be accountable for all aspects of the work.

Content licence: This is an Open Access article distributed in accordance with the terms of the Creative Commons Attribution (CC BY-NC-ND 4.0) licence, which permits use, distribution and reproduction in any medium, provided that the original publication is properly cited, the use is noncommercial (i.e., research or educational use), and no modifications or adaptations are made. See: https://creativecommons. org/licenses/by-nc-nd/4.0/

Funding: Bassel Al Wattar holds a personal Academic Clinical Lectureship from the UK National Health Institute of Research. Khalid
Khan is a Distinguished Investigator funded by the Beatriz Galindo (senior modality) Program Grant given to the University of Granada by the Ministry of Science, Innovation, and Universities of the Spanish Government.

Copyright: This is an Open Access article distributed in accordance with the terms of the Creative Commons Attribution (CC BY 4.0) license, which permits others to distribute, remix, adapt and build upon this work, for commercial use, provided the original work is properly cited. See: http://creativecommons. org/licenses/by/4.0/.

Data sharing: All data included in this study is available to other researchers for use in scientific research. Requests should be made to the corresponding authors stating the purpose and type of data required.

Accepted: Jan. 18, 2021

Correspondence to: Bassel Al Wattar, dr. basselwa@gmail.com 